Cahiers québécois de démographie

\title{
Les inégalités socio-économiques et culturelles de la mortalité à Montréal à la fin des années 1980
}

THE SOCIO-ECONOMIC AND CULTURAL INEQUALITIES IN

MONTREAL'S DEATH RATE AT THE END OF THE 1980'S

DESIGUALDADES SOCIOECONOMICAS Y CULTURALES DE MORTALIDAD EN MONTREAL A FINES DE LOS ANOS 1980

\section{Robert Choinière}

Volume 22, numéro 2, automne 1993

URI : https://id.erudit.org/iderudit/010151ar

DOI : https://doi.org/10.7202/010151ar

Aller au sommaire du numéro

Éditeur(s)

Association des démographes du Québec

ISSN

0380-1721 (imprimé)

1705-1495 (numérique)

Découvrir la revue

Citer cet article

Choinière, R. (1993). Les inégalités socio-économiques et culturelles de la mortalité à Montréal à la fin des années 1980. Cahiers québécois de démographie, 22(2), 339-362. https://doi.org/10.7202/010151ar
Résumé de l'article

Cette étude traite des inégalités économiques et culturelles de la mortalité à partir d'une approche écologique à l'échelle des secteurs de recensement. La proportion de personnes pauvres, estimée à partir du revenu et de la taille des ménages privés, a été utilisée comme mesure du niveau socio-économique et la proportion d'immigrants comme mesure du statut ethno-culturel Nous avons attribué à chaque décès la proportion de pauvres et le pourcentage d'immigrants observés dans le secteur de résidence du décédé au recensement de 1986. Les décès ont été classés en quintiles et ternies selon différents revenus et en quintiles d'immigrants. Les résultats montrent que des écarts importants subsistent à Montréal selon le revenu et que l'on observe aussi des disparités importantes selon le quintile d'immigrants et selon le croisement des tertiles de revenu et d'immigrants. 
Cahiers québécois de démographie

Vol. 22, no 2, automne 1993, p. 339-362.

\title{
Les inégalités socio-économiques et culturelles de la mortalité à Montréal à la fin des années 1980
}

\author{
Robert CHOINIËRE *
}

Cet article constitue la suite d'un texte sur les disparités géographiques de la mortalité dans la région de Montréal publié précédemment dans les Cahiers québécois de démographie (Choinière, 1991). Les résultats obtenus indiquaient que malgré une baisse importante de la mortalité dans la région montréalaise, l'on observait des écarts importants entre certains territoires de centres locaux de services communautaires (CLSC). En appliquant les méthodes de la régression linéaire, nous montrions qu'ensemble la proportion de personnes pauvres et le pourcentage d'immigrants expliquaient plus de $80 \%$ des variations de l'espérance de vie d'un CLSC à l'autre. Les durées de vie les plus longues se trouvaient dans les CLSC présentant à la fois les plus faibles proportions de personnes pauvres et les pourcentages d'immigrants les plus élevés.

La présente étude a pour objectif d'examiner les inégalités face à la mort sur le territoire de l'île de Montréal à partir des données sur le revenu et sur les immigrants observées à l'échelle des secteurs de recensement. Elle vise à démontrer que la mortalité baisse à mesure que la proportion de personnes vivant sous le seuil de faible revenu diminue et que la proportion d'immigrants augmente. Par rapport à l'étude précédente, qui analysait les données par territoire de CLSC, ici, les individus sont classés en quintiles et en tertiles d'après les

\footnotetext{
Direction de la santé publique de Montréal-Centre. Une version préliminaire de cet article a été présentée au Colloque de l'Association des démographes du Québec dans le cadre du congrès annuel de l'Association canadiennefrançaise pour l'avancement des sciences tenu à Montréal en mai 1992.
} 
caractéristiques de leur secteur de recensement de résidence. De plus, l'analyse des inégalités ne se limite pas à la mortalité générale, elle couvre certaines causes prépondérantes de décès.

\section{MÉTHODOLOGE}

Les décès sont répartis selon la proportion de personnes vivant sous le seuil de faible revenu et le pourcentage d'immigrants ${ }^{1}$ observés en 1986 à l'échelle du secteur de recensement de résidence du décédé. Les seuils de faibles revenus sont établis en fonction du revenu et de la taille des ménages privés. Par exemple, le seuil de faible revenu est de 10233 dollars pour les individus vivant seuls, et de 29155 dollars pour les membres d'un ménage de sept personnes ou plus. Le seuil de faible revenu est souvent utilisé pour identifier les personnes pauvres.

Le secteur de recensement est une unité géographique comprenant de 2500 à 8000 habitants, et dont les limites correspondent à des traits physiques permanents. Les secteurs de recensement doivent être le plus homogènes possible sur le plan des caractéristiques économiques et sociales de leur population (Statistique Canada, 1992a : 229). En attribuant à chaque décès les proportions de personnes et d'immigrants obtenues à l'échelle du secteur de résidence du décédé, on suppose que les personnes pauvres se retrouvent principalement dans des secteurs présentant des taux élevés de pauvreté et que les immigrants se concentrent dans les secteurs ayant des pourcentages élevés d'immigrants. Jusqu'à maintenant, cette méthodologie a été utilisée uniquement pour étudier la mortalité et les naissances selon le revenu à l'échelle de l'ensemble des régions urbaines du Canada (Wilkins, Adams et Brancker, 1989; Wilkins, Sherman et Best, 1991; Ugnat et Mark, 1987; Wigle et Mao, 1980).

Le secteur de recensement de résidence du décédé n'apparaît pas sur l'acte de décès. Cependant, à partir du code postal, il est possible de retracer le secteur de recensement en utilisant le fichier de conversion de Statistique Canada, qui indique, pour chacun des 44000 codes postaux de l'île de Montréal, le

1 Le pays de naissance apparait sur l'acte de décès. Le taux de réponse est de l'ordre de $85 \%$ en 1990. Pour les besoins de cette êtude, nous nous devions d'utiliser la mêrne méthodologie pour répartir les décès selon différentes catégories. 
secteur de recensement correspondant. La même opération a été effectuée pour les naissances, qui servent de dénominateur dans le calcul du taux de mortalité infantile.

Pour $95 \%$ des dêcès et $89 \%$ des naissances, le code postal de résidence était indiqué sur l'acte de décès ou de naissance. Il était ainsi possible d'attribuer aux décédés les proportions de personnes pauvres et d'immigrants observées dans le secteur de recensement de résidence. Dans les cas où le code postal était incomplet, nous avons attribué les proportions observées à l'échelle des régions de tri d'acheminement (RTA) qui correspondent aux trois premières positions du code postal. Statistique Canada fournit pour les RTA les mêmes informations que celles qui sont contenues dans les profils par secteur de recensement. Enfin, dernière mesure, si les trois premières positions du code postal étaient absentes sur les actes, nous nous sommes basé sur les pourcentages de pauvres ou d'immigrants observés à l'échelle de la municipalité de résidence.

Une fois ces étapes franchies, nous avons utilisé les proportions de pauvres et d'immigrants pour répartir les décès en quintiles de revenu et d'immigrants. Ces quintiles ont été déterminés selon l'effectif de population de chaque secteur plutôt que d'après le nombre de secteurs. L'on retrouve ainsi, dans chaque quintile, près de $20 \%$ de la population de l'île de Montréal (tableau 1).

Finalement, pour tenir compte à la fois des personnes pauvres et des immigrants, nous avons divisé chacune de ces deux variables en tertiles de façon à ce que le croisement des tertiles forme neuf groupes. Le tableau 1 montre la constitution de ces neuf groupes.

La période couverte par l'étude comprend cinq années, 1986 à $1990^{2}$. Ce sont les seules années pour lesquelles le code postal au complet apparait sur les actes de décès ou de naissance. Le dénominateur utilisé dans le calcul des indices de mortalité est la population recensée en 1986.

La méthodologie employée dans cette étude peut présenter certains biais. Ainsi, nous associons aux décédés les caractéristiques observées à l'échelle des secteurs de recensement. De plus, les données sur les personnes pauvres et les immigrants excluent la population vivant en institution, alors que les

2 Ni le fichier des décès pour l'année 1991 ni les données du recensement de 1991 sur la population immigrante et le revenu n'étaient disponibles au moment où cette étude a été réalisée. 
TABLEAU 1 - Constitution des quintiles et des tertiles de revenu et d'immigrants à partir des proportions observées dans les secteurs de recensement, île de Montréal, 1986

\begin{tabular}{|c|c|c|c|}
\hline \multicolumn{4}{|c|}{ QUINTILES DE REVENU } \\
\hline Quintile & $\begin{array}{c}\text { Proportion de } \\
\text { pauures (\%) }\end{array}$ & Population & $\begin{array}{c}\text { Décès } \\
1986-1990\end{array}$ \\
\hline Faible & 38,22 et plus & 351880 & 17725 \\
\hline Moyen faible & 27,79 à 38,21 & 353640 & 17172 \\
\hline Moyen & 20,39 à 27,78 & 353600 & 15715 \\
\hline Moyen élevé & 14,42 à 20,38 & 342025 & 13438 \\
\hline Élevé & moins de 14,42 & 346825 & 12326 \\
\hline \multicolumn{4}{|c|}{ QUINTILES D'IMMIGRANTS } \\
\hline Quintile & $\begin{array}{l}\text { Proportion d'im- } \\
\text { migrants (\%) }\end{array}$ & Population & $\begin{array}{c}\text { Décès } \\
1986-1990\end{array}$ \\
\hline Faible & Moins de 8,41 & 342225 & 17568 \\
\hline Moyen faible & 8,41 à 15,75 & 357440 & 18278 \\
\hline Moyen & 15,76 à 23,95 & 345960 & 13089 \\
\hline Moyen élevé & 23,96 à 33,28 & 350960 & 12813 \\
\hline Élevé & 33,29 et plus & 351385 & 14628 \\
\hline
\end{tabular}

CROISEMENT DES TERTILES DE REVENU ET D' IMMIGRANTS

\begin{tabular}{|c|c|c|c|c|c|}
\hline $\begin{array}{l}\text { Tertile } \\
\text { de } \\
\text { revenu }\end{array}$ & $\begin{array}{l}\text { Tertile } \\
\text { d'immi- } \\
\text { grants }\end{array}$ & $\begin{array}{c}\text { Proportion } \\
\text { de pauvres } \\
\text { (\%) }\end{array}$ & $\begin{array}{c}\text { Proportion } \\
\text { d'immigrants } \\
(\%)\end{array}$ & $\begin{array}{l}\text { Popu- } \\
\text { lation }\end{array}$ & $\begin{array}{l}\text { Décès } \\
1986- \\
1990\end{array}$ \\
\hline Faible & Faible & 31,01 et plus & Moins de 13,93 & 190375 & 7781 \\
\hline Faible & Moyen & 31,01 et plus & 13,93 à 26,58 & 292775 & 9337 \\
\hline Faible & Élevé & 31,01 et plus & 26,59 et plus & 97575 & 4575 \\
\hline Moyen & Faible & 18,80 à 31,00 & Moins de 13,93 & 163250 & 6487 \\
\hline Moyen & Moyen & 18,80 à 31,00 & 13,93 à 26,58 & 207020 & 9147 \\
\hline Moyen & Élevé & 18,80 à 31,00 & 26,59 et plus & 211385 & 10127 \\
\hline Élevé & Faible & Moins de 18,60 & Moins de 13,93 & 215095 & 8770 \\
\hline Élevé & Moyen & Moins de 18,60 & 13,93 à 26,58 & 98610 & 4694 \\
\hline Élevé & Élevé & Moins de 18,60 & 26,59 et plus & 271885 & 15458 \\
\hline
\end{tabular}


mesures de mortalité utilisées couvrent l'ensemble de la population. Nous faisons ainsi l'hypothèse que les proportions de pauvres ou d'immigrants sont les mêmes parmi les pensionnaires d'institutions que dans le reste de la population. Enfin, les résultats peuvent être affectés par la concentration géographique des institutions. La concentration des institutions hospitalières dans les secteurs de recensement les plus pauvres pourrait, par exemple, accroître artificiellement les inégalités entre les quintiles de revenu.

Cependant, en l'absence d'informations sur l'acte de décès, la méthodologie employée dans cette étude demeure la plus précise pour mesurer la mortalité selon certaines caractéristiques. De plus, comme nous l'avons signalé, les secteurs sont des unités territoriales qui présentent en principe des caractéristiques économiques et sociales homogènes (Statistique Canada, 1992a : 229). L'on peut ainsi penser que les caractéristiques du secteur fournissent dans la plupart des cas un portrait fidèle des individus qui y résident.

\section{LA MORTALITÉ SELON LE gUINTILE DE REVENU}

Les résultats obtenus pour l'espérance de vie à la naissance et le taux de mortalité infantile selon le quintile de revenu montrent que la mortalité évolue selon un gradient (tableaux 2 , et 3). L'espérance de vie augmente et la mortalité infantile diminue à mesure que le quintile de revenu s'élève. Les écarts de l'espérance de vie entre les quintiles élevé et faible sont plus marqués chez les hommes $(6,1$ ans) que chez les femmes; ( 2,4 ans). L'écart entre les hommes et les femmes est de près de 9 ans pour le quintile le plus faible et d'un peu plus de 5 ans dans le groupe le plus riche. Le taux de mortalité infantile est de $70 \%$ plus élevé dans le groupe le plus pauvre par rapport au groupe le plus favorisé.

En général, l'on retrouve la même relation entre le revenu et la mortalité dans les différents groupes d'âge (tableau 4). Certains groupes dérogent à ce constat. Chez les femmes de 1-14 ans ainsi que chez celles de 75 ans et plus, c'est le quintile de revenu le plus élevé qui présente les plus hauts indices de mortalité. Chez les garçons de 1-14 ans, le quintile le plus défavorisé enregistre les taux de mortalité les plus bas.

Le tableau 5 présente les indices comparatifs de mortalité selon le quintile de revenu pour certaines causes prépondérantes de décès. Pour la plupart des causes, la mortalité 
TABLEAU 2 - Espérance de vie à la naissance, selon le quintile de revenu et le sexe, île de Montréal, 1986-1990 (en années)

\begin{tabular}{lccc}
\hline $\begin{array}{l}\text { Quintile } \\
\text { de revenu }\end{array}$ & Hommes & Femmes & $\begin{array}{c}\text { Écart hommes- } \\
\text { femmes }\end{array}$ \\
\hline Faible & 69,4 & 78,2 & 8,8 \\
Moyen faible & 70,9 & 79,0 & 8,1 \\
Moyen & 72,4 & 79,5 & 7,1 \\
Moyen élevé & 73,3 & 80,0 & 6,7 \\
Élevé & 75,5 & 80,6 & 5,1 \\
Total & 72,2 & 79,5 & 7,3 \\
Écart élevé-faible & 6,1 & 2,4 & \\
\hline
\end{tabular}

Sources : MSSS, fichiers des décès et des naissances, 1986 à 1990. Statistique Canada, recensement de 1986 .

augmente avec la pauvreté. Les disparités semblent plus marquées chez les hommes que chez les femmes. L'on observe chez les premiers que pour le sida, les suicides, les traumatismes en général, les maladies pulmonaires, les maladies de l'appareil respiratoire en général, le cancer du poumon et les maladies vasculaires, le groupe le plus pauvre enregistre une mortalité d'au moins $50 \%$ plus élevée que celle du groupe le plus riche.

Chez les femmes, les différences les plus importantes entre quintiles faible et élevé sont observées pour les suicides, les maladies pulmonaires et le cancer du poumon. Pour plusieurs causes, les femmes qui sont dans le groupe le plus riche présentent une mortalité plus élevée que celles des groupes les plus pauvres. C'est le cas notamment des femmes décédant du cancer du sein, de maladies de l'appareil respiratoire, de pneumonie et grippe ou des suites d'accidents de la route.

\section{LA MORTALITÉ SELON LE gUINTILE D'IMMIGRANTS}

L'espérance de vie tend à augmenter à mesure que le quintile d'immigrants s'élève (tableau 6). L'on remarque cependant que la durée de vie est légèrement plus longue dans le quintile moyen élevé que dans le plus élevê. L'écart entre les quintiles faible et élevé est de 4,5 ans chez les hommes et de 2,5 ans chez les femmes. Comme pour le revenu, les différences de longévité entre les hommes et les femmes diminuent lorsque l'on passe du quintile le plus bas au quintile le plus élevé.

Les résultats obtenus à partir du taux de mortalité infantile diffèrent beaucoup de ceux qui sont observés pour l'espérance de vie. Le tableau 7 montre que les taux les plus élevés se 
TABLEAU 3 - Taux de mortalité infantile, selon le quintile de revenu, île de Montréal, 1986-1990 (taux pour 1000 naissances)

\begin{tabular}{lcc}
\hline Quintile de revenu & Taux & Indice $^{\text {a }}$ \\
\hline Faible & 9,6 & 171,4 \\
Moyen faible & 7,6 & 135,7 \\
Moyen & 6,8 & 121,4 \\
Moyen élevé & 5,7 & 101,8 \\
Élevé & 5,6 & 100,0 \\
Total & 7,1 & \\
\hline
\end{tabular}

Source : MSSS, fichiers des décès et des naissances, 1986 à 1990.

a. Calculé par rapport au quintile le plus êlevé, qui est fixé à 100.

TABLEAU 4 - Indice comparatif de mortalité a selon le quintile de revenu, par groupe d'âge et par sexe, île de Montréal, 1986-1990

\begin{tabular}{|c|c|c|c|c|c|}
\hline \multirow[b]{2}{*}{ Groupe d'âge } & \multicolumn{5}{|c|}{ Quintile de revenu } \\
\hline & Faible & $\begin{array}{l}\text { Moyen } \\
\text { faible }\end{array}$ & Moyen & $\begin{array}{c}\text { Moyen } \\
\text { élevé }\end{array}$ & Élevé \\
\hline & \multicolumn{5}{|c|}{ HOMMES } \\
\hline Moins d'un an & 175,4 & 141,4 & 116,2 & 105,4 & 100,0 \\
\hline $1-14$ ans & 92,5 & 173,6 & 178,9 & 123,7 & 100,0 \\
\hline $15-24$ ans & 145,8 & 143,6 & 134,6 & 156,4 & 100,0 \\
\hline 25-44 ans & 237,2 & 189,3 & 148,2 & 124,3 & 100,0 \\
\hline 45-64 ans & 201,7 & 176,3 & 145,2 & 124,3 & 100,0 \\
\hline 65-74 ans & 156,0 & 138,2 & 130,1 & 125,2 & 100,0 \\
\hline 75 ans et plus & 115,7 & 109,2 & 108,2 & 106,8 & 100,0 \\
\hline \multirow[t]{2}{*}{ Total } & 151,7 & 136,3 & 124,6 & 117,1 & 100,0 \\
\hline & \multicolumn{5}{|c|}{ FEMMES } \\
\hline Moins d'un an & 167,0 & 130,8 & 130,6 & 96,5 & 100,0 \\
\hline $1-14$ ans & 84,5 & 86,2 & 73,7 & 79,3 & 100,0 \\
\hline $15-24$ ans & 170,7 & 171,4 & 186,3 & 153,6 & 100,0 \\
\hline 25-44 ans & 150,3 & 131,2 & 125,6 & 105,3 & 100,0 \\
\hline 45-64 ans & 160,4 & 137,9 & 126,6 & 110,0 & 100,0 \\
\hline $65-74$ ans & 138,1 & 118,8 & 112,0 & 112,4 & 100,0 \\
\hline 75 ans et plus & 94,0 & 98,9 & 95,3 & 93,9 & 100,0 \\
\hline Total & 113,0 & 109,1 & 103,8 & 100,0 & 100,0 \\
\hline
\end{tabular}

Sources : MSSS, fichier des décès, 1986 à 1990. Statistique Canada, recensement de 1986. a. On calcule l'indice en rapportant pour chaque groupe d'âge le taux de chaque quintile à
celui du quintile le plus élevé, qui est fixé à 100. Pour le total, les taux ont été ajustés
selon la structure par àge et par sexe de la population de l'île de Montréal en 1986 . 
TABLEAU 5 - Indice comparatif de mortalité a , selon le quintile de revenu, par cause de décès et par sexe, île de Montréal, 1986-1990

\begin{tabular}{|c|c|c|c|c|c|}
\hline \multirow[b]{2}{*}{ Cause } & \multicolumn{5}{|c|}{ Quintile de revenu } \\
\hline & Faible & $\begin{array}{l}\text { Moyen } \\
\text { faible }\end{array}$ & Moyen & $\begin{array}{l}\text { Moyen } \\
\text { élevé }\end{array}$ & Élevé \\
\hline & \multicolumn{5}{|c|}{ HoMmes } \\
\hline Cancers & 141,0 & 131,1 & 127,1 & 120,8 & 100,0 \\
\hline Poumon & 199,3 & 183,0 & 166,9 & 144,2 & 100,0 \\
\hline Côlon et rectum & 105,2 & 120,6 & 122,2 & 112,2 & 100,0 \\
\hline Prostate & 109,3 & 97,2 & 104,2 & 120,4 & 100,0 \\
\hline $\begin{array}{l}\text { Appareil circulatoire } \\
\text { Cardiopathies }\end{array}$ & 139,0 & 129,8 & 122,6 & 115,4 & 100,0 \\
\hline ischémiques & 138,5 & 132,0 & 121,8 & 114,1 & 100,0 \\
\hline Maladies vasculaires & 158,6 & 145,0 & 145,6 & 135,8 & 100,0 \\
\hline Appareil respiratoire & 165,4 & 138,5 & 122,9 & 115,7 & 100,0 \\
\hline Pneumonie et grippe & 109,9 & 86,1 & 80,6 & 81,9 & 100,0 \\
\hline Maladies pulmonaires & 203,6 & 182,0 & 146,5 & 136,2 & 100,0 \\
\hline Traumatismes & 196,2 & 168,1 & 155,4 & 136,9 & 100,0 \\
\hline Accidents de la route & 101,0 & 113,5 & 125,9 & 106,3 & 100,0 \\
\hline Suicides & 238,5 & 200,6 & 176,9 & 148,1 & 100,0 \\
\hline \multirow[t]{2}{*}{ Sida } & 290,3 & 398,8 & 173,9 & 120,1 & 100,0 \\
\hline & \multicolumn{5}{|c|}{ FEMMES } \\
\hline Cancers & 101,6 & 100,9 & 100,8 & 103,7 & 100,0 \\
\hline Poumon & 130,5 & 111,7 & 105,3 & 110,7 & 100,0 \\
\hline Côlon et rectum & 121,5 & 137,7 & 140,7 & 120,1 & 100,0 \\
\hline Sein & 86,5 & 92,7 & 94,0 & 110,1 & 100,0 \\
\hline Appareil circulatoire & 120,4 & 115,8 & 113,2 & 104,2 & 100,0 \\
\hline $\begin{array}{l}\text { Cardiopathies } \\
\text { ischémiques }\end{array}$ & 1251 & 1237 & 125 & 1006 & \\
\hline Maladies vasculaires & 110,2 & 109,6 & 102,7 & 95,1 & 100,0 \\
\hline Appareil respiratoire & 76,3 & 73,8 & 56,3 & 62,7 & 100,0 \\
\hline Pneumonie et grippe & 85,2 & 89,2 & 74,5 & 79,4 & 100,0 \\
\hline Maladies pulmonaires & 138,4 & 131,3 & 92,0 & 99,7 & 100,0 \\
\hline Traumatismes & 122,1 & 116,7 & 98,4 & 84,9 & 100,0 \\
\hline Accidents de la route & 68,4 & 117,7 & 76,6 & 110,8 & 100,0 \\
\hline Suicides & 147,8 & 136,4 & 92,4 & 97,9 & 100,0 \\
\hline
\end{tabular}

Source : MSSS, fichier des décès, 1986 à 1990 .

a. On calcule l'indice en rapportant pour chaque cause le taux ajusté de chaque quintile à celui du quintile le plus êlevé, qui est fixé à 100. Les taux ont été ajustés selon la structure par âge et par sexe de la population de l'île de Montréal en 1986. 
TABLEAU 6 - Espérance de vie à la naissance, selon le quintile d'immigrants et le sexe, île de Montréal, 1986-1990 (en années)

\begin{tabular}{lccc}
\hline Quintile d'immigrants & Hommes & Femmes & Écart hommes-femmes \\
\hline Faible & 69,5 & 78,0 & 8,5 \\
Moyen faible & 71,0 & 78,6 & 7,6 \\
Moyen & 72,8 & 79,9 & 7,1 \\
Moyen élevé & 74,2 & 80,7 & 6,4 \\
Élevé & 74,0 & 80,5 & 6,5 \\
Total & 72,2 & 79,5 & 7,3 \\
Écart élevé-faible & 4,5 & 2,5 & \\
\hline
\end{tabular}

Sources : MSSS, fichiers des décès et des naissances, 1986 à 1990. Statistique Canada, recensement de 1986 .

retrouvent dans le groupe ayant la plus forte proportion d'immigrants tandis que les taux les plus faibles sont enregistrés pour les quintiles moyen et moyen élevé.

Lorsque l'on examine les différences entre quintiles d'immigrants selon l'âge et le sexe (tableau 8), à l'exception des moins d'un an chez les hommes et des 15-24 ans chez les femmes, les quintiles ayant le moins d'immigrants présentent une surmortalité par rapport aux quintiles comptant de fortes proportions d'immigrants.

Pour la plupart des causes retenues, la mortalité tend à augmenter à mesure que le quintile d'immigrants diminue (tableau 9). Comme pour le revenu, les différences entre les quintiles d'immigrants sont plus marquées chez les hommes que chez les femmes. Chez les hommes, la mortalité du quintile le plus bas est d'au moins $50 \%$ plus élevée que celle du quintile le plus élevé pour le cancer en général, le cancer du poumon, les cardiopathies ischémiques, les maladies pulmo-

TABLEAU 7 - Taux de mortalité infantile, selon le quintile d'immigrants, île de Montréal, 1986-1990 (taux pour 1000 naissances)

\begin{tabular}{lcc}
\hline Quintile d'immigrants & Taux & Indice $^{\text {a }}$ \\
\hline Faible & 7,6 & 96,2 \\
Moyen faible & 7,7 & 97,5 \\
Moyen & 6,1 & 77,2 \\
Moyen élevé & 6,1 & 77,2 \\
Elevé & 7,9 & 100,0 \\
Total & 7,1 & \\
\hline
\end{tabular}

Source : MSSS, fichiers des décès et des naissances, 1986 à 1990 .

a. Calculé par rapport au quintile le plus élevé, qui est fixé à 100 . 
TABLEAU 8 - Indice comparatif de mortalité a , selon le quintile d'immigrants, par groupe d'äge et par sexe, île de Montréal, 1986-1990

\begin{tabular}{|c|c|c|c|c|c|}
\hline \multirow[b]{2}{*}{ Groupe d'âge } & \multicolumn{5}{|c|}{ Quintile d'immigrants } \\
\hline & Faible & $\begin{array}{c}\text { Moyen } \\
\text { faible }\end{array}$ & Moyen & $\begin{array}{c}\text { Moyen } \\
\text { élevé }\end{array}$ & Élevé \\
\hline & \multicolumn{5}{|c|}{ HOMMES } \\
\hline Moins d'un an & 95,7 & 91,5 & 70,6 & 72,0 & 100,0 \\
\hline $1-14$ ans & 145,1 & 131,1 & 114,4 & 100,8 & 100,0 \\
\hline $15-24$ ans & 131,2 & 137,0 & 99,6 & 80,3 & 100,0 \\
\hline 25-44 ans & 138,1 & 120,5 & 95,9 & 81,9 & 100,0 \\
\hline 45-64 ans & 161,8 & 134,7 & 105,2 & 100,1 & 100,0 \\
\hline $65-74$ ans & 154,9 & 137,8 & 122,7 & 105,5 & 100,0 \\
\hline 75 ans et plus & 133,1 & 128,5 & 121,2 & 104,3 & 100,0 \\
\hline \multirow[t]{2}{*}{ Total } & 126,4 & 122,5 & 91,1 & 89,6 & 100,0 \\
\hline & \multicolumn{5}{|c|}{ FEMMES } \\
\hline Moins d'un an & 95,9 & 105,4 & 87,8 & 86,3 & 100,0 \\
\hline $1-14$ ans & 125,9 & 118,5 & 106,3 & 98,3 & 100,0 \\
\hline $15-24$ ans & 98,2 & 109,0 & 85,7 & 80,1 & 100,0 \\
\hline $25-44$ ans & 122,5 & 128,0 & 86,9 & 90,8 & 100,0 \\
\hline $45-64$ ans & 143,9 & 120,1 & 105,4 & 98,4 & 100,0 \\
\hline $65-74$ ans & 144,9 & 125,2 & 116,6 & 106,2 & 100,0 \\
\hline 75 ans et plus & 117,5 & 116,2 & 105,5 & 97,2 & 100,0 \\
\hline Total & 119,9 & 123,3 & 90,8 & 85,7 & 100,0 \\
\hline
\end{tabular}

Sources : MSSS, fichier des décès, 1986 à 1990. Statistique Canada, recensement de 1986.

a. On calcule l'indice en rapportant pour chaque groupe d'âge le taux de chaque quintile à celui du quintile le plus élevé, qui est fixé à 100. Pour le total, les taux ont été ajustés selon la structure par âge et par sexe de la population de l'ile de Montréal en 1986.

naires, les traumatismes en général et les suicides. Chez les femmes, c'est seulement pour le cancer du poumon que l'on observe un écart aussi important entre les quintiles extrêmes.

\section{LA MORTALITÉ SELON LE CROISEMENT DES TERTILES DE REVENU ET D'IMMIGRANTS}

Le croisement des tertiles de revenu et d'immigrants montre que l'espérance de vie la plus élevée est enregistrée par la population se situant à la fois dans le plus haut tertile de revenu et dans le plus haut tertile d'immigrants (tableau 10). À 
TABLEAU 9-Indice comparatif de mortalité a, selon le quintile d'immigrants, par cause de décès et par sexe, ûle de Montréal, 1986-1990

\begin{tabular}{|c|c|c|c|c|c|}
\hline \multirow[b]{2}{*}{ Cause } & \multicolumn{5}{|c|}{ Quintile d'immigrants } \\
\hline & Faible & $\begin{array}{l}\text { Moyen } \\
\text { faible }\end{array}$ & Moyen & $\begin{array}{c}\text { Moyen } \\
\text { élevé }\end{array}$ & Élevé \\
\hline & \multicolumn{5}{|c|}{ HOMMES } \\
\hline Cancers & 155,2 & 139,4 & 124,5 & 113,8 & 100,0 \\
\hline Poumon & 199,1 & 172,9 & 136,4 & 124,0 & 100,0 \\
\hline Côlon et rectum & 145,4 & 137,7 & 103,3 & 111,2 & 100,0 \\
\hline Prostate & 136,7 & 135,9 & 128,3 & 122,1 & 100,0 \\
\hline Appareil circulatoire & 139,4 & 129,5 & 115,9 & 99,5 & 100,0 \\
\hline $\begin{array}{l}\text { Cardiopathies } \\
\text { ischémiques }\end{array}$ & 147,9 & 131,7 & 115,8 & 103,2 & 100,0 \\
\hline Maladies vasculaires & 120,1 & 131,3 & 108,9 & 93,5 & 100,0 \\
\hline Appareil respiratoire & 80,7 & 143,3 & 126,0 & 99,7 & 100,0 \\
\hline Pneumonie et grippe & 117,6 & 102,7 & 106,2 & 97,6 & 100,0 \\
\hline Maladies pulmonaires & 207,4 & 184,2 & 149,8 & 104,0 & 100,0 \\
\hline Traumatismes & 154,0 & 134,0 & 111,4 & 98,1 & 100,0 \\
\hline Accidents de la route & 138,2 & 112,3 & 136,4 & 89,4 & 100,0 \\
\hline Suicides & 182,2 & 162,3 & 104,3 & 109,4 & 100,0 \\
\hline \multirow[t]{2}{*}{ Sida } & 126,4 & 127,3 & 71,0 & 57,4 & 100,0 \\
\hline & \multicolumn{5}{|c|}{ FEMMES } \\
\hline Cancers & 119,2 & 112,4 & 107,4 & 105,3 & 100,0 \\
\hline Poumon & 150,0 & 116,5 & 105,9 & 100,8 & 100,0 \\
\hline Côlon et rectum & 122,2 & 147,5 & 106,4 & 93,1 & 100,0 \\
\hline Sein & 116,8 & 113,3 & 109,9 & 109,9 & 100,0 \\
\hline Appareil circulatoire & 136,2 & 131,1 & 116,0 & 101,2 & 100,0 \\
\hline Cardiopathies & & & & & \\
\hline $\begin{array}{l}\text { 1schemiques } \\
\text { Maladies vasculaires }\end{array}$ & $\begin{array}{l}144,5 \\
121,9\end{array}$ & $\begin{array}{l}142,2 \\
122,7\end{array}$ & $\begin{array}{l}119,8 \\
119,6\end{array}$ & $\begin{array}{l}102,3 \\
104,2\end{array}$ & $\begin{array}{l}100,0 \\
100,0\end{array}$ \\
\hline Appareil respiratoire & 111,3 & 115,8 & 96,0 & 95,4 & 100,0 \\
\hline Pneumonie et grippe & 117,5 & 101,6 & 82,0 & 92,3 & 100,0 \\
\hline Maladies pulmonaires & 145,6 & 136,3 & 112,2 & 94,9 & 100,0 \\
\hline Traumatismes & 109,8 & 123,6 & 93,9 & 87,9 & 100,0 \\
\hline Accidents de la route & 91,3 & 116,9 & 101,7 & 80,4 & 100,0 \\
\hline Suicides & 127,6 & 139,0 & 89,2 & 114,6 & 100,0 \\
\hline
\end{tabular}

Source : MSSS, fichier des décès, 1986 à 1990 .

a. On calcule l'indice en rapportant pour chaque cause le taux ajusté de chaque quintile à celui du quintile le plus élevé, qui est fixé à 100. Les taux ont été ajustés selon la structure par àge et par sexe de la population de l'île de de Montréal en 1986. 
TABLEAU 10 - Espérance de vie à la naissance, selon le tertile de revenu et le tertile d'immigrants, par sexe, île de Montréal, 1986-1990

\begin{tabular}{|c|c|c|c|c|c|}
\hline \multirow{2}{*}{$\begin{array}{l}\text { Tertile de } \\
\text { revenu }\end{array}$} & \multicolumn{5}{|c|}{ Tertile d'immigrants } \\
\hline & Faible & Moyen & Élevé & Total & $\begin{array}{c}\text { Ecart élevé } \\
\text { faible }\end{array}$ \\
\hline & \multicolumn{5}{|c|}{ HOMMES } \\
\hline Faible & 68,6 & 69,4 & 72,3 & 70,0 & 3,7 \\
\hline Moyen & 71,4 & 72,5 & 73,8 & 72,4 & 2,4 \\
\hline Élevé & 71,5 & 74,9 & 75,4 & 74,5 & 3,9 \\
\hline Total & 70,1 & 73,1 & 73,8 & 72,2 & 3,7 \\
\hline \multirow[t]{2}{*}{ Écart êlevé-faible } & 2,9 & 5,5 & 3,1 & 4,5 & \\
\hline & \multicolumn{5}{|c|}{ FEMMES } \\
\hline Faible & 77,8 & 77,8 & 78,1 & 78,6 & 0,3 \\
\hline Moyen & 78,7 & 80,0 & 80,7 & 79,6 & 2,0 \\
\hline Élevé & 78,1 & 80,7 & 80,9 & 80,3 & 2,8 \\
\hline Total & 78,2 & 79,9 & 80,4 & 79,5 & 2,2 \\
\hline Écart èlevé-faible & 0,3 & 2,9 & 2,8 & 1,7 & \\
\hline
\end{tabular}

Sources : MSSS, fichier des décès, 1986 à 1990. Statistique Canada, recensement de 1986.

l'inverse, la durée de vie la plus courte est enregistrée dans le groupe compris dans les tertiles de revenu et d'immigrants les plus faibles. L'écart entre les valeurs extrêmes est de 6,8 ans chez les hommes et de 3,1 ans chez les femmes.

En général, quel que soit le tertile d'immigrants, l'espérance de vie augmente en fonction du tertile de revenu. De même, quel que soit le tertile de revenu, la durẻe de vie s'allonge à mesure que le tertile d'immigrants s'élève. C'est pour le tertile d'immigrants moyen que l'écart entre les tertiles de revenu est le plus élevé, et pour le tertile de revenu le plus élevé que l'écart entre les tertiles d'immigrants est le plus important.

Le tableau 11 montre que le taux de mortalité infantile le plus bas se retrouve dans le groupe ayant les tertiles de revenu et d'immigrants les plus élevés, et le taux le plus haut dans le groupe se situant dans le tertile de revenu faible et le tertile d'immigrants moyen. La mortalité infantile diminue à mesure que le tertile de revenu augmente, peu importe le tertile d'immigrants. C'est à l'intérieur du tertile d'immigrants le plus élevé que les inégalités de la mortalité infantile selon le revenu sont les plus importantes. L'on n'observe pas de tendance de la 
TABLEAU 11 - Taux de mortalité infantile, selon le tertile de revenu et le tertile d'immigrants, ille de Montréal, 1986-1990

\begin{tabular}{lccccc}
\hline Tertile de & \multicolumn{4}{c}{ Tertile d'immigrants } \\
revenu & Faible & Moyen & Élevé & Total & $\begin{array}{c}\text { Écart élevé- } \\
\text { faible }\end{array}$ \\
\hline Faible & 7,8 & 9,8 & 9,0 & 8,6 & $-1,2$ \\
Moyen & 7,7 & 6,6 & 6,9 & 7,1 & 0,8 \\
Ėlevé & 5,2 & 6,1 & 4,9 & 5,6 & 0,3 \\
Total & 7,3 & 6,9 & 7,2 & 7,1 & 0,1 \\
Écart faible-élevé & 2,6 & 3,7 & 4,1 & & \\
\hline
\end{tabular}

Source : MSSS, fichiers des décès et des naissances, 1986 à 1990.

mortalité infantile selon le tertile d'immigrants, quel que soit le tertile de revenu retenu.

Le tableau 12 présente les indices comparatifs de mortalité selon le croisement des tertiles de revenu et d'immigrants pour chacun des deux sexes par groupe d'âge. Les indices sont calculés par rapport à la catégorie présentant à la fois les tertiles de revenu et d'immigrants les plus élevés. Pour ce groupe, l'indice est fixé à 100 .

Chez les garçons et les filles âgés de 1 à 14 ans, le croisement des tertiles de revenu et d'immigrants ne permet de dégager aucune tendance de la mortalité. Chez les hommes de moins d'un an et de 45-64 ans, de même que chez les femmes de moins d'un an, de 15-24 ans et de 25-44 ans, les inégalités de mortalitê sont plus marquées selon le tertile de revenu que selon le tertile d'immigrants. À l'inverse, la mortalité tend plus à varier selon le tertile d'immigrants chez les hommes de 75 ans et plus et chez les femmes de 65 ans et plus. Dans les autres groupes d'âge, la mortalité semble varier autant selon le tertile de revenu que selon le tertile d'immigrants.

Pour tous les groupes d'âge, à l'exception des 1-14 ans chez les hommes et chez les femmes, les indices les plus élevés se retrouvent dans le tertile de revenu le plus faible ou dans le tertile d'immigrants le plus faible. Chez les hommes et les femmes de 25 ans à 74 ans, les indices les plus élevés sont observés dans le groupe ayant à la fois les tertiles de revenu et d'immigrants les plus faibles.

L'on retrouve au tableau 13 les résultats du croisement des tertiles de revenu et d'immigrants pour les causes prépondérantes de décès. À l'intérieur de chacun des tertiles de revenu, 


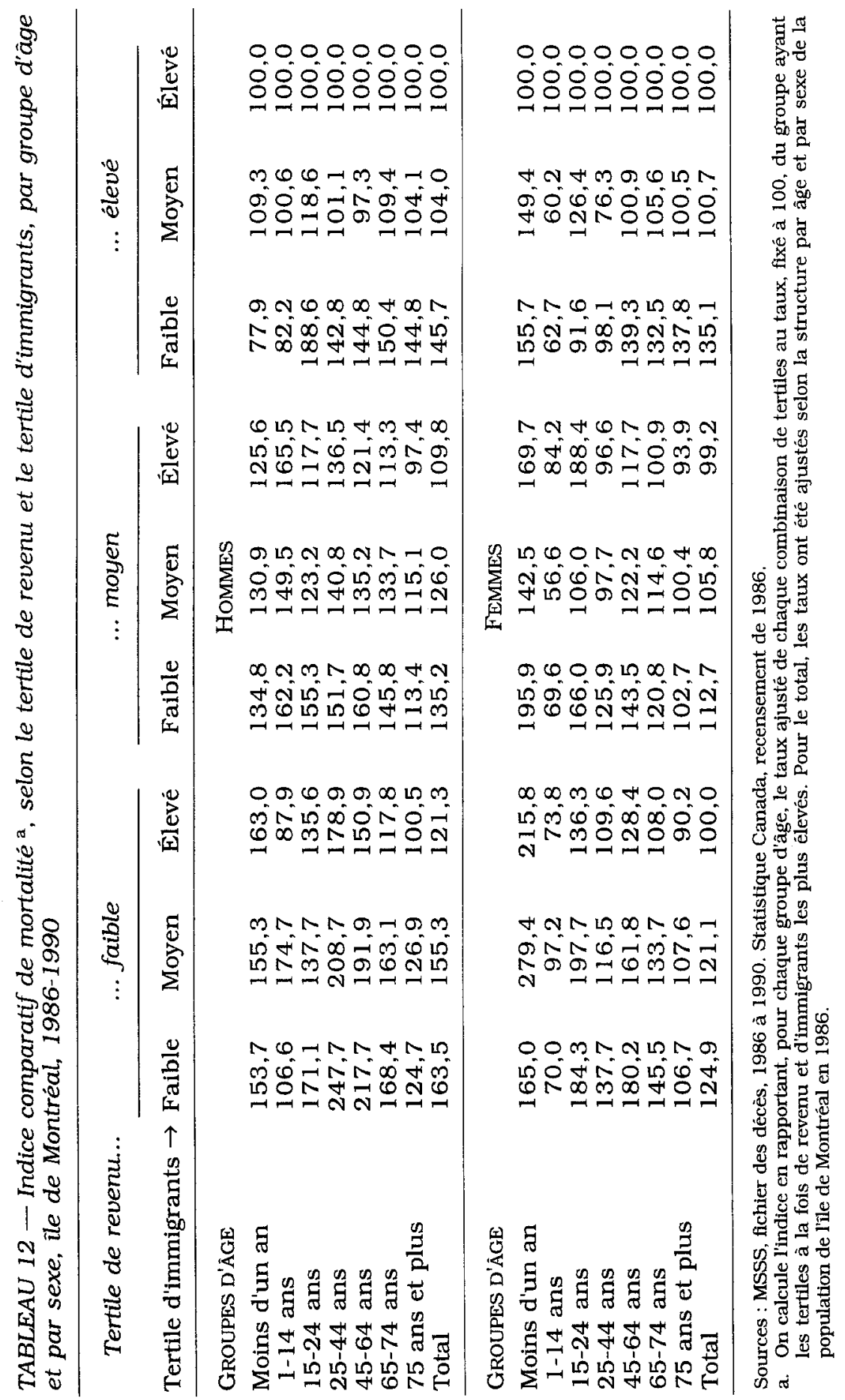


la mortalité selon le tertile d'immigrants suit un gradient chez les hommes pour le cancer en général, le cancer du poumon, les maladies de l'appareil circulatoire en général, les maladies de l'appareil respiratoire en général, les maladies pulmonaires et les accidents de la route, et chez les femmes pour les maladies de l'appareil circulatoire en général, les cardiopathies ischémiques et les maladies pulmonaires.

À l'inverse, lorsque l'on isole le tertile d'immigrants, la mortalité n'augmente en fonction du tertile de revenu pour aucune cause chez les femmes et augmente pour les traumatismes en général, les suicides et le sida chez les hommes.

Pour toutes les causes, sauf pour le cancer du côlon et du rectum chez les femmes, les indices de mortalité les plus élevés sont enregistrés soit dans le tertile de revenu le plus faible, soit dans le tertile d'immigrants le plus faible. Pour dix causes sur quatorze chez les hommes et quatre causes sur treize chez les femmes, la mortalité la plus forte se retrouve dans le groupe formé à la fois des tertiles de revenu et d'immigrants les plus faibles. Les disparités les plus importantes sont observées chez les hommes, en particulier pour le sida, les maladies pulmonaires, les suicides et le cancer du poumon.

\section{DISCUSSION}

Les personnes appartenant à un quintile de revenu faible vivent en moyenne 6,1 ans de moins si elles sont de sexe masculin et 2,4 ans de moins si elles sont de sexe féminin que les personnes comprises dans le quintile le plus élevé (tableau 14). Wilkins, Adams et Brancker (1989) obtiennent pour l'ensemble des régions urbaines du Canada en 1986 un écart de 5,7 ans chez les hommes et de 1,8 an chez les femmes entre les quintiles de revenu extrêmes.

En ce qui concerne les quintiles d'immigrants, les différences entre les catégories "faible" et "élevé" sont de 4,5 ans chez les hommes et de 2,5 ans chez les femmes. Enfin, lorsque l'on croise les tertiles de revenu et d'immigrants, les écarts sont de 6,8 ans pour les hommes et de 3,1 ans chez les femmes entre la population appartenant à la fois aux tertiles de revenu et d'immigrants les plus élevés et la population des tertiles les plus faibles. De plus, lorsque l'on isole le tertile d'immigrants, l'espérance de vie augmente selon le tertile de revenu, et, à l'inverse, quel que soit le tertile de revenu, la durée de vie s'allonge en fonction du tertile d'immigrants. 


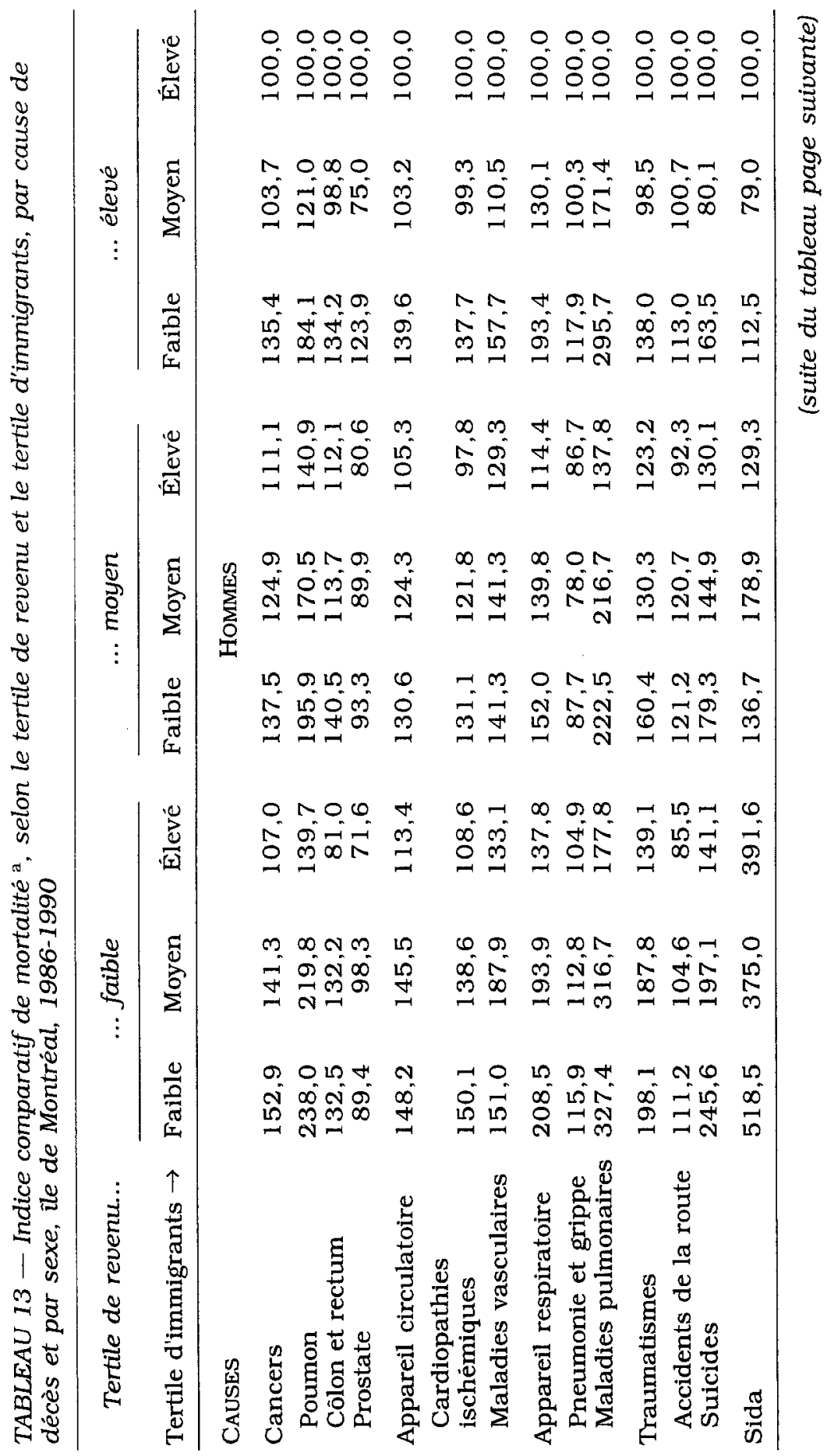




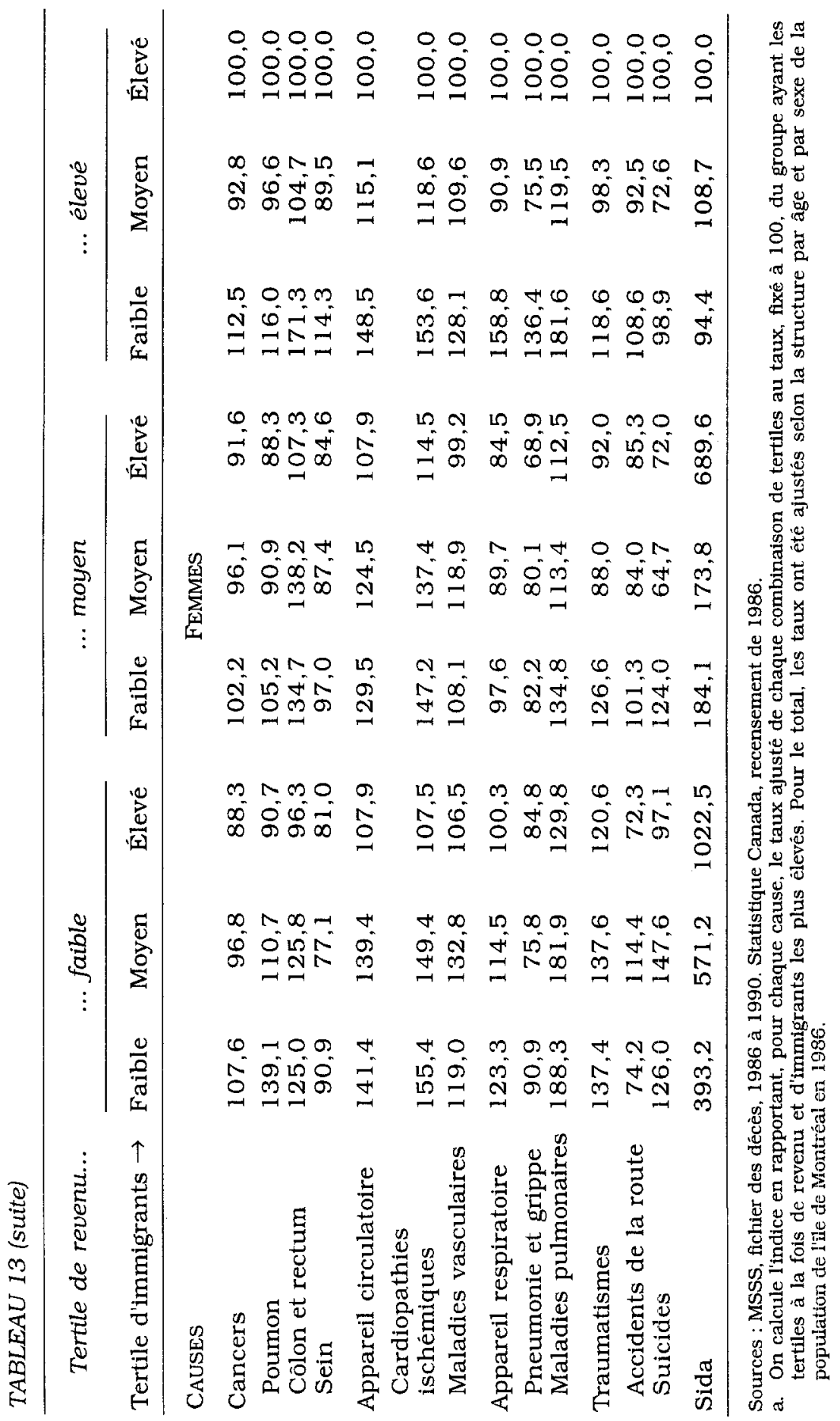


TABLEAU 14 - Synthèse des résultats pour l'espérance de vie à la naissance et le taux de mortalité infantile

\begin{tabular}{|c|c|c|c|}
\hline & \multicolumn{2}{|c|}{$\begin{array}{l}\text { Espérance de vie à la } \\
\text { naissance (en années) }\end{array}$} & \multirow{2}{*}{$\begin{array}{l}\text { Taux de morta- } \\
\text { lité infantile } \\
\text { (pour } 1000 \\
\text { naissances) }\end{array}$} \\
\hline & Hommes & Femmes & \\
\hline \multicolumn{4}{|l|}{ Quintile de revenu } \\
\hline Quintile le plus faible & 69,4 & 78,2 & 9,6 \\
\hline Quintile le plus élevé & 75,5 & 80,6 & 5,6 \\
\hline Écart & 6,1 & 2,4 & 4,0 \\
\hline \multicolumn{4}{|l|}{ Quintile d'immigrants } \\
\hline Quintile le plus faible & 69,5 & 78,0 & 7,6 \\
\hline Quintile le plus élevé & 74,0 & 80,5 & 7,9 \\
\hline Écart & 4,5 & 2,5 & $-0,3$ \\
\hline \multicolumn{4}{|l|}{$\begin{array}{l}\text { Croisement des tertiles de } \\
\text { revenu et d'immigrants }\end{array}$} \\
\hline $\begin{array}{l}\text { Tertiles de revenu et } \\
\text { d'immigrants faibles }\end{array}$ & 68,6 & 77,8 & 7,8 \\
\hline $\begin{array}{l}\text { Tertiles de revenu et } \\
\text { d'immigrants élevés }\end{array}$ & 75,4 & 80,9 & 4,9 \\
\hline Écart & 6,8 & 3,1 & 2,9 \\
\hline
\end{tabular}

Sources : tableaux 2, 3, 6, 7, 10 et 11.

Le taux de mortalitê infantile évolue selon un gradient en ce qui concerne les tertiles de revenu mais pas pour les tertiles d'immigrants. La mortalité tend à augmenter à mesure que le tertile d'immigrants diminue à partir du groupe d'âge 1-14 ans chez les hommes et du groupe 25-44 ans chez les femmes. Ces résultats corroborent les hypothèses que nous faisions dans notre précédente étude (Choinière, 1991). La sélection positive des immigrants, qui expliquerait la durée de vie plus longue du quintile d'immigrants le plus élevé, ne serait pas un facteur important pour les groupes d'âge les plus jeunes. Pour la mortalité infantile, l'accès aux services de santé et aux programmes de soins prénatals et post-natals et l'exposition aux facteurs de risque seraient plus déterminants que la sélection positive des parents lors de leur arrivée. L'on ne peut parler dans ce dernier cas de sélection positive des enfants puisqu'il s'agit d'enfants décédés avant l'âge d'un an. Ainsi, la probabilité qu'un enfant de moins d'un an ayant immigré au Québec décède par la suite avant d'atteindre son premier anniversaire doit être très faible. 
Les caractéristiques de la population immigrante (lieu de provenance, niveau socio-économique) varient selon l'âge. La provenance des immigrants a considérablement évolué. Les Européens représentaient $90 \%$ des immigrants arrivés au Canada avant 1961 mais seulement $25 \%$ des immigrants arrivés entre 1981 et 1991 (Statistique Canada, 1992b). Au Québec, la plupart des immigrants arrivés en 1990 venaient de pays du Tiers Monde; $54 \%$ étaient nés en Asie, $17 \%$ en Europe, $18 \%$ en Amérique et $11 \%$ en Afrique (Duchesne, 1992).

D'une période à l'autre, plus de $65 \%$ des immigrants qui ont pour destination le Québec ont moins de 30 ans lors de leur arrivée au Canada (Choinière et Robitaille, 1990). L'on a aussi observé que plus la principale période d'immigration des groupes ethniques autres que français et britannique était ancienne, plus ces groupes présentaient une structure par âge vieille et des conditions socio-économiques favorables (Choinière, Robitaille et Bourbeau, 1987).

L'on peut donc penser que dans les groupes d'âge les plus jeunes, la population immigrante est constituée d'individus arrivés durant les dernières années et présentant des conditions socio-économiques moins favorables que les immigrants des groupes d'âge plus avancés. Cela expliquerait pourquoi, aux jeunes âges, la mortalité ne semble pas diminuer selon le quintile d'immigrants, contrairement à ce qui se passe aux âges élevés, où la population immigrante aurait une mortalité inférieure à celle du reste de la population.

Étant donné que la mortalité se concentre dans les derniers groupes d'âge, soit dans les groupes où les immigrants présenteraient des conditions socio-économiques plus favorables que le reste de la population, l'on ne sera pas étonné de constater qu'en général les indices de mortalité selon la cause diminuent à mesure que le quintile d'immigrants augmente.

Le tableau 15 présente une synthèse des indices comparatifs de mortalité selon la cause pour les groupes extrêmes. Dans presque tous les cas, les différences de mortalité sont plus importantes chez les hommes que chez les femmes.

Chez ces dernières, sauf pour les traumatismes en général et les suicides, les inégalités de la mortalité sont plus grandes selon le quintile d'immigrants que selon le quintile de revenu. L'on observe une surmortalité supérieure à $40 \%$ entre les quintiles de revenu faible et élevé pour les suicides, entre les quintiles d'immigrants faible et élevé pour le cancer du poumon, les cardiopathies ischémiques et les maladies pulmo- 
TABLEAU 15 - Indices comparatifs de mortalité : synthèse des résultats

\begin{tabular}{|c|c|c|c|c|c|c|}
\hline \multirow[b]{2}{*}{ Causes } & \multicolumn{2}{|c|}{$\begin{array}{l}\text { Quintile de } \\
\text { revenu }\end{array}$} & \multicolumn{2}{|c|}{$\begin{array}{c}\text { Quintile } \\
\text { d'immigrants }\end{array}$} & \multicolumn{2}{|c|}{$\begin{array}{l}\text { Tertiles de revenu } \\
\text { et d'immigrants }\end{array}$} \\
\hline & Faible & Élevé & Faible & Élevé & Faible & Élevé \\
\hline & \multicolumn{6}{|c|}{ HOMMES } \\
\hline Cancers & 141,0 & 100,0 & 155,2 & 100,0 & 152,9 & 100,0 \\
\hline Poumon & 199,3 & 100,0 & 199,1 & 100,0 & 238,0 & 100,0 \\
\hline Côlon et rectum & 105,2 & 100,0 & 145,4 & 100,0 & 132,5 & 100,0 \\
\hline Prostate & 109,3 & 100,0 & 136,7 & 100,0 & 89,4 & 100,0 \\
\hline Appareil circulatoire & 139,0 & 100,0 & 139,4 & 100,0 & 148,2 & 100,0 \\
\hline $\begin{array}{l}\text { Cardiopathies } \\
\text { ischémiques }\end{array}$ & 138,5 & 100,0 & 147,9 & 100,0 & 150,1 & 100,0 \\
\hline $\begin{array}{l}\text { Maladies } \\
\text { vasculaires }\end{array}$ & 158,6 & 100,0 & 120,1 & 100,0 & 151,0 & 100,0 \\
\hline Appareil respiratoire & 165,4 & 100,0 & 80,7 & 100,0 & 208,5 & 100,0 \\
\hline $\begin{array}{l}\text { Pneumonie } \\
\text { et grippe }\end{array}$ & 109,9 & 100,0 & 117,6 & 100,0 & 115,9 & 100,0 \\
\hline $\begin{array}{l}\text { Maladies } \\
\text { pulmonaires }\end{array}$ & 203,6 & 100,0 & 207,4 & 100,0 & 327,4 & 100,0 \\
\hline Traumatismes & 196,2 & 100,0 & 154,0 & 100,0 & 198,1 & 100,0 \\
\hline $\begin{array}{l}\text { Accidents de } \\
\text { la route }\end{array}$ & 101,0 & 100,0 & 138,2 & 100,0 & 111,2 & 100,0 \\
\hline Suicides & 238,5 & 100,0 & 182,2 & 100,0 & 245,6 & 100,0 \\
\hline \multirow[t]{2}{*}{ Sida } & 290,3 & 100,0 & 126,4 & 100,0 & 518,5 & 100,0 \\
\hline & \multicolumn{6}{|c|}{ FEMMES } \\
\hline Cancers & 101,6 & 100,0 & 119,2 & 100,0 & 107,6 & 100,0 \\
\hline Poumon & 130,5 & 100,0 & 150,0 & 100,0 & 139,1 & 100,0 \\
\hline Côlon et rectum & 121,5 & 100,0 & 122,2 & 100,0 & 125,0 & 100,0 \\
\hline Sein & 86,5 & 100,0 & 116,8 & 100,0 & 90,9 & 100,0 \\
\hline Appareil circulatoire & 120,4 & 100,0 & 136,2 & 100,0 & 141,4 & 100,0 \\
\hline $\begin{array}{l}\text { Cardiopathies } \\
\text { ischémiques }\end{array}$ & 125,1 & 100,0 & 144,5 & 100,0 & 155,4 & 100,0 \\
\hline $\begin{array}{l}\text { Maladies } \\
\text { vasculaires }\end{array}$ & 110,2 & 100,0 & 121,9 & 100,0 & 119,0 & 100,0 \\
\hline Appareil respiratoire & 76,3 & 100,0 & 111,3 & 100,0 & 123,3 & 100,0 \\
\hline $\begin{array}{l}\text { Pneumonie } \\
\text { et grippe }\end{array}$ & 85,2 & 100,0 & 117,5 & 100,0 & 90,9 & 100,0 \\
\hline $\begin{array}{l}\text { Maladies } \\
\text { pulmonaires }\end{array}$ & 138,4 & 100,0 & 145,6 & 100,0 & 188,3 & 100,0 \\
\hline Traumatismes & 122,1 & 100.0 & 109,8 & 100,0 & 137,4 & 100,0 \\
\hline $\begin{array}{l}\text { Accidents de } \\
\text { la route }\end{array}$ & 68,4 & 100,0 & 91,3 & 100,0 & 74,2 & 100,0 \\
\hline Suicides & 147,8 & 100,0 & 127,6 & 100,0 & 126,0 & 100,0 \\
\hline
\end{tabular}

Sources : tableaux 5,9 et 13 . 
naires, et entre les tertiles de revenu et d'immigrants faible et élevé pour les maladies de l'appareil circulatoire en général, les cardiopathies ischémiques et les maladies pulmonaires.

Chez les hommes, les écarts de mortalité sont plus importants selon le quintile de revenu pour le cancer du poumon, les maladies vasculaires, les maladies de l'appareil respiratoire, les traumatismes en général, les suicides et le sida. À l'opposé, l'on observe des disparités plus importantes entre les tertiles de revenu et d'immigrants extrêmes pour le cancer en général, le cancer du côlon et du rectum, le cancer de la prostate, les cardiopathies ischémiques, les pneumonies et grippes et les accidents de la route. Pour certaines causes, la mortalité du groupe le plus défavorisé est au moins deux fois supérieure à celle du groupe le plus favorisé. C'est le cas pour le cancer du poumon et les maladies pulmonaires, quel que soit le quintile ou le tertile, les maladies de l'appareil respiratoire pour les quintiles de revenu, ainsi que les traumatismes en général, les suicides et le sida pour les quintiles de revenu et les tertiles de revenu et d'immigrants.

Dans certains cas, les disparités sont très faibles. Dans d'autres, l'on observe une mortalité plus faible dans le groupe le plus vulnérable. Chez les hommes, pour le cancer de la prostate selon les tertiles de revenu et d'immigrants et pour les maladies de l'appareil respiratoire selon le quintile d'immigrants, le groupe le plus favorisé présente une surmortalité par rapport au groupe le plus défavorisé. Le même phénomène se produit chez les femmes pour le cancer du sein et les pneumonies et grippes selon le quintile de revenu et le croisement des tertiles de revenu et d'immigrants, pour les maladies de l'appareil respiratoire selon le quintile de revenu, et pour les accidents de la route selon les trois statuts. Wilkins, Adams et Brancker (1989) obtiennent des résultats semblables pour la mortalité selon le quintile de revenu chez les femmes en ce qui concerne le cancer du sein, les pneumonies et les accidents de la route.

La surmortalité des pauvres (quintile de revenu le plus faible) par rapport aux riches (quintile de revenu le plus élevé) pourrait s'expliquer par des habitudes de vie, des comportements préventifs et un environnement social différents. Ainsi, selon les données de l'Enquête Santé Québec de 1987 (Colin, Lavoie et Poulin, 1989), les personnes socio-économiquement défavorisées présenteraient, par rapport au reste de la population, une plus forte proportion de fumeurs, une plus grande 
prévalence de personnes obèses, un indice de saines habitudes de vie plus faible, un plus haut pourcentage de personnes ayant connu des événements stressants, un indice de santé globale moins favorable, une fréquence plus grande des incapacités sévères, un état de santé mentale plus précaire, plus d'idées suicidaires et un plus haut niveau de détresse psychologique.

Ces résultats vont de pair avec les indices élevés de mortalité observés pour le quintile de revenu le plus faible en ce qui concerne le cancer du poumon, les maladies de l'appareil circulatoire, les maladies pulmonaires et les suicides.

Guant au sida, on peut se demander si les disparités importantes observées selon le quintile de revenu ne sont pas le résultat de la maladie : pour cette cause, on trouve 30 décès, auxquels est associé le même code postal, classés dans le quintile de revenu le plus faible. La localisation dans les secteurs les plus pauvres des institutions traitant les personnes atteintes du sida qui sont dans la dernière phase de la maladie pourrait ainsi gonfler artificiellement la surmortalité par sida dans ce groupe. La concentration des communautés homosexuelles dans des quartiers défavorisés pourrait aussi expliquer en partie ces résultats.

En ce qui a trait aux différences observées selon le croisement des tertiles de revenu et d'immigrants, comme il a été mentionné plus haut, elles pourraient s'expliquer en partie par la sélection positive des immigrants et leur situation socioéconomique favorable eu égard au reste de la population dans les groupes d'âge où se concentrent les décès. L'Enquête de 1987 décèle peu de différences entre les communautés culturelles et les Québécois d'origine en ce qui concerne les habitudes de vie et l'environnement social (Cousineau, 1989).

Le fait de diviser la population en neuf groupes à partir du croisement des tertiles de revenu et d'immigrants a pour effet d'accentuer les inégalités face à la mort puisque l'on retient dans le groupe présentant les indices de mortalité les plus faibles les secteurs ayant à la fois les plus faibles proportions de pauvres et les plus fortes proportions d'immigrants. 


\section{REFÉRENCES BIBLIOGRAPHIQUES}

CHOINIĖRE, Robert, 1991. "Les disparités géographiques de la mortalité dans le Montréal métropolitain, 1984-1988 : étude écologique des liens avec les conditions sociales, économiques et culturelles", Cahiers québécois de démographie, 20, $1: 115-144$.

CHOINIËRE, Robert, et Norbert ROBITAILLE, 1990. "The Aging of Ethnic Groups in Quebec", dans S. HALLI, F. TROVATO et L. DRIEDGER, éd. Ethnic Demography: Canadian Immigrant, Racial and Cultural Variations. Ottawa, Carleton University Press : 253-271.

CHOINIĖRE, Robert, Norbert ROBITAILLE et Robert BOURBEAU, 1987. "Aperçu des minorités ethniques du Québec", Apprentissage et socialisation, 10, 2:81-86.

COLIN, Christine, Jean-Pierre LAVOIE et Carole POULIN, 1989. Les Personnes défavorisées. Québec, Les Publications du Québec, coll. "Et la santé, ça va ?", no 3, 119 p.

COUSINEAU, Daniel, 1989. Les Communautés culturelles. Québec, Les Publications du Guébec, coll. "Et la santé, ça va ?", no 5, 54 p.

DUCHESNE, Louis, 1992. La Situation démographique au Québec. Édition 1991-1992. Québec, Les Publications du Québec, 231 p.

STATISTIQUE CANADA, 1992a. Dictionnaire du recensement de 1991. Ottawa, no 92-301F au catalogue, $386 \mathrm{p}$.

STATISTIQUE CANADA, 1992b. Le Quotidien. Ottawa, 8 décembre : 6-7.

UGNAT, A. M., et E. MARK, 1987. "L'espérance de vie selon le sexe, l'âge et le revenu", Maladies chroniques au Canada, 8, 1: 12-13.

WIGLE, D. T., et Y. MAO, 1980. Mortalité urbaine au Canada selon le niveau de revenu. Ottawa, Laboratoire de lutte contre la maladie.

WILKINS, Russell, Gregory J. SHERMAN et P. A. F. BEST, 1991. "Issues de grossesse et mortalité infantile selon le revenu dans les régions urbaines du Canada en 1986", Rapports sur la santé, 3, 1 : 7-34.

WILKINS, Russell, Owen ADAMS et Anna BRANCKER, 1989. «Évolution de la mortalité selon le revenu dans les régions urbaines du Canada entre 1971 et 1986", Rapports sur la santé, I, 2 : 137-174. 


\section{RÉSUMÉ - SUMMARY - RESUMEN}

CHOINIĖRE Robert - LES INÉGALITÉS SOCIO-ÉCONOMIQUES ET CULTURELLES DE LA MORTALTÉ À MONTRÉAL À LA FIN DES ANNÉES 1980

Cette étude traite des inégalités économiques et culturelles de la mortalité à partir d'une approche écologique à l'échelle des secteurs de recensement. La proportion de personnes pauvres, estimée à partir du revenu et de la taille des ménages privés, a été utilisée comme mesure du niveau socio-économique et la proportion d'immigrants comme mesure du statut ethno-culturel. Nous avons attribué à chaque décès la proportion de pauvres et le pourcentage d'immigrants observés dans le secteur de résidence du décédé au recensement de 1986. Les décès ont été classés en quintiles et tertiles selon différents revenus et en quintiles d'immigrants. Les résultats montrent que des écarts importants subsistent à Montréal selon le revenu et que l'on observe aussi des disparités importantes selon le quintile d'immigrants et selon le croisement des tertiles de revenu et d'immigrants.

CHOINIÈRE Robert - THE SOCIO-ECONOMIC AND CULTURAL INEQUALITIES IN MONTREAL'S DEATH RATE AT THE END OF THE 1980'S

This study examines the socio-economic and cultural inequalities of the mortality rate using an ecological approach at the census tract level. The proportion of poor individuals, as estimated from the income and size of private households, was used as a measure of socio-economic status, while the proportion of immigrants was used as a measure of cultural status. Each death was classified in relation to the proportion of poor and the percentage of immigrants found in the residential sector of the dead according to the 1986 census statistics. The results show that substantial differences exist in Montreal based on income; and significant disparities were also found according to the quintile of immigrants and the combination of income and immigrant tertiles.

\section{CHOINIÈRE Robert - DESIGUALDADES SOCIOECONÓMICAS Y CULTURALES} DE MORTALIDAD EN MONTREAL A FINES DE LOS AÑOS 1980

El presente estudio trata de las desigualdades económicas $y$ culturales de mortalidad desde un enfoque ecológico a escala de los sectores de censo. Se utilizó la proporción de personas pobres, estimada a partir del ingreso y del tamaño de las familias, como medida del nivel socioeconómico, y la proporción de inmigrantes como medida del estatuto etno-cultural. Se atribuyó a cada defunción la proporción de pobres $y$ el porcentaje de inmigrantes observados en el sector de residencia del fallecido según el censo de 1986. Se clasificaron las defunciones en quintiles $y$ tertiles según distintos ingresos, $y$ en quintiles de inmigrantes. Los resultados muestran que siguen existiendo diferencias importantes en Montreal según el ingreso, y se observan asimismo desniveles notables según el quintil de inmigrantes y según la combinación de los tertiles de ingreso y de inmigrantes. 